\title{
Diffuse Interstellar Bands in Emission
}

\author{
T. B. Williams ${ }^{1,2}$, P. Sarre ${ }^{3}$, C. C. M. Marshall ${ }^{3}$, K. Spekkens ${ }^{4}$, and \\ R. Kuzio de Naray ${ }^{5}$
}

${ }^{1}$ South African Astronomical Observatory,

PO Box 9, Observatory 7935, South Africa

email: williams@saao.ac.za

${ }^{2}$ Dept. of Physics \& Astronomy, Rutgers, The State University of New Jersey, 136 Frelinghuysen Rd., Piscataway NJ 08854 USA

${ }^{3}$ School of Chemistry, The University of Nottingham,

University Park, Nottingham NG7 2RD, UK

email: peter.sarre@nottingham.ac.uk

${ }^{4}$ Dept. of Physics, Royal Military College of Canada,

PO Box 17000, Station Forces, Kingston ON K7K 7B4, XNS Canada

email: kristine.spekkens@rmc.ca

${ }^{5}$ Dept. of Physics and Astronomy, Georgia State University,

25 Park Place, Atlanta GA 30303 USA

email: kuzio@astro.gsu.edu

\begin{abstract}
Recent Fabry-Pérot observations towards the galaxy NGC 1325 with the Southern African Large Telescope (SALT) led to the serendipitous discovery of an emission feature centered at $661.3 \mathrm{~nm}$ arising from material in the interstellar medium (ISM) of our Galaxy; this emission feature lies at the wavelength of one of the sharper and stronger diffuse bands normally seen in absorption. The flux of the feature is $4.2 \pm 0.5 \times 10^{-18} \mathrm{e} \mathrm{s}^{-1} \mathrm{~cm}^{-2} \operatorname{arcsec}^{-2}$. It appears that this is the first observation of emission from a diffuse band carrier in the ISM, excited in this case by the interstellar radiation field. We present the discovery spectra and describe follow-up measurements proposed for SALT.
\end{abstract}

Keywords. ISM: lines and bands, ISM: molecules

Introduction. The longest-standing problem in astronomical spectroscopy is the identification of the carriers of the diffuse interstellar absorption bands (DIBs), the first examples of which were discovered on photographic plates over 80 years ago. Most researchers consider a population of large carbon-based molecules to be responsible for the DIBs (e.g. Léger \& d'Hendecourt 1985, $A \mathscr{G} A$ 146, 81). Identification of the carriers would open a new probe of interstellar conditions and processes in interstellar clouds and could have implications far beyond - including the role of such molecules in star and planet formation and even for the origins of life. Only one clear-cut example exists where complementary emission (from a subset) of DIBs is seen - in the Red Rectangle nebula - where the emission is excited by radiation from the central star HD 44179 (Scarrott et al.2012, MNRAS 255, 11p).

Observations. We used the Fabry-Pérot system of the Robert Stobie Spectrograph on the Southern African Large Telescope to measure the $\mathrm{H} \alpha$ kinematics of nearby galaxies for the RINGS survey (Mitchell et al.2015, AJ 149, 116). The spectral resolution of each image is $0.4 \mathrm{~nm} \mathrm{FWHM}$, and there is a $2.4 \mathrm{~nm}$ radial wavelength gradient across the 8 ' field of view. Uniform discrete spectral features thus produce rings in the images. Measuring the night-sky background in galaxy-subtracted images revealed an unidentified spectral feature at $661.3 \mathrm{~nm}$, corresponding to the wavelength of a strong DIB. The feature is present in the field toward NGC $1325\left(l=212^{\circ}, b=-54^{\circ}\right)$, but absent toward NGC $2280\left(l=237^{\circ}, b=-13^{\circ}\right)$ observed on the same nights. On each of the two observing nights, the feature is detected on all 9 images of the NGC 1325 field that contain its wavelength. Fig. 1 shows sample images and spectra and Table 1 lists the characteristics of the emission. 

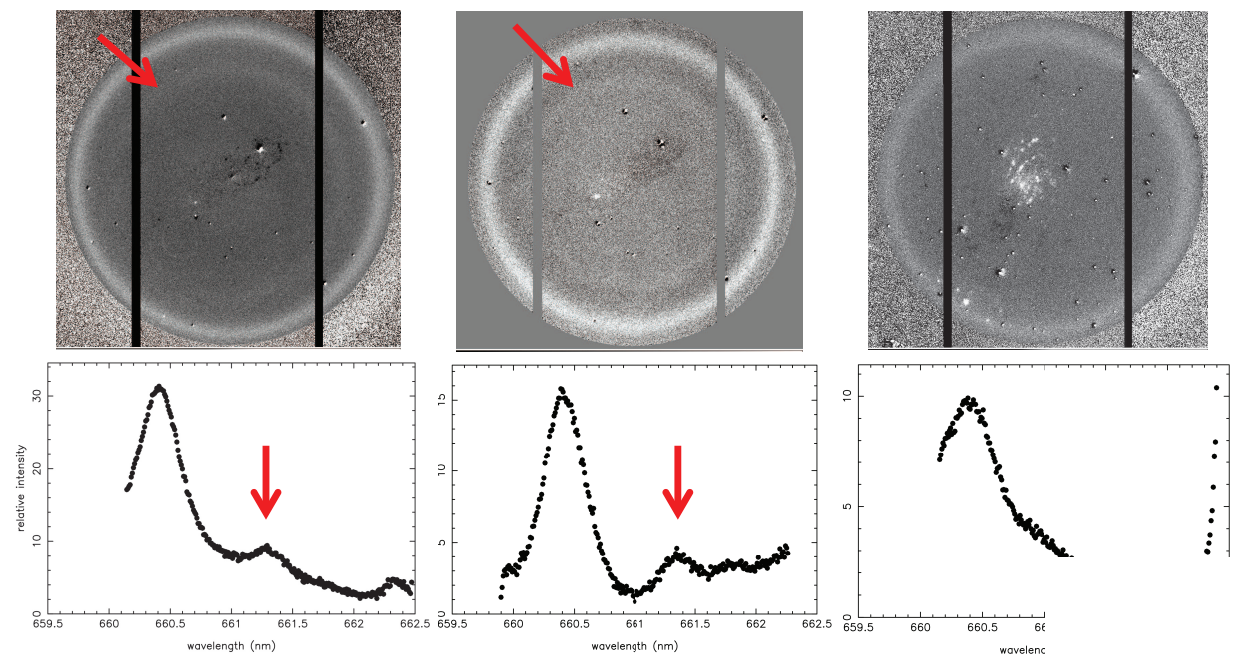

Figure 1. Median-subtracted images and azimuthally-averaged spectra. Left: NGC 1325 on 01 Nov. 2011; Center: NGC 1325 on 28 Dec. 2011; Right: NGC 2280 on 01 Nov. 2011. Arrow indicates the $661.3 \mathrm{~nm}$ emission feature.

Table 1. Emission feature in the field of NGC 1325

\begin{tabular}{|c|c|c|c|}
\hline $\begin{array}{c}\text { Date } \\
2011\end{array}$ & $\left(\mathrm{e} \mathrm{s}^{-1} \mathrm{~cm}^{-2} \operatorname{arc-sec}^{-2}\right)$ & $\begin{array}{c}\text { Observed Wavelength } \\
(\mathrm{nm})\end{array}$ & $\begin{array}{c}\text { Heliocentric Wavelength } \\
(\mathrm{nm})\end{array}$ \\
\hline $01 \mathrm{Nov}$ & $4.03 \pm 0.62 \times 10^{-18}$ & $661.33 \pm 0.01$ & $661.33 \pm 0.01$ \\
\hline $28 \mathrm{Dec}$ & $4.29 \pm 0.17 \times 10^{-18}$ & $661.38 \pm 0.02$ & $661.34 \pm 0.02$ \\
\hline
\end{tabular}

Discussion. There are no known night sky emission features at $661.3 \mathrm{~nm}$ (Osterbrock et al.1996, PASP 108, 227). The feature is detected with the same flux, within the uncertainties, on both nights of observation, while no emission is detected toward NGC 2280 on the same nights. Finally the observed wavelength of the feature differs significantly on the two nights, but after correction to the heliocentric frame the wavelengths agree within the uncertainties. All these considerations argue strongly that the feature is not of terrestrial origin, but arises along the line of sight toward NGC 1325. The constant wavelength and uniform distribution over the field of view clearly show that the emission is not associated with NGC 1325 itself. We thus speculate that this emission arises in the interstellar medium of our Galaxy along the line of sight toward NGC 1325. The wavelength coincidence with one of the strong DIBs suggests that this emission is produced by the same (unknown) carrier that produces DIB absorption. We plan additional SALT observations to search for emission from other strong DIB features toward NGC 1325, and also in the vicinity of the Red Rectangle and other likely targets. If the association with DIBs is confirmed, these sorts of measurements can provide additional information to address the long-standing question of the source of the DIBs. 